\title{
Agrupación de estudiantes según rendimiento académico: ¿Afecta el núcleo pedagógico?
}

\section{Ability Grouping: the pedagogical core is changed?}

ISSN 1510-2432 - ISSN 1688-9304 (en línea) - DOI: http://dx.doi.org/10.18861/cied.2016.7.1.2578

María Paola Sevilla

Magister en Políticas Educativas, Universidad Alberto Hurtado, Chile. Master en Economía, Georgetown University. Estudiante, Programa de Doctorado en Educación, Universidad Alberto Hurtado-Diego Portales, Chile.

Fecha de recibido: 26/09/2015

Fecha de aceptado: 01/03/2016

\section{Resumen}

Este artículo reflexiona sobre las prácticas de agrupación de estudiantes al interior de las escuelas y su nexo con los resultados de aprendizaje. Se realiza una revisión bibliográfica que contrasta distintas posiciones y evidencia empírica existente en torno a la agrupación de estudiantes según habilidad -tracking-. Se plantea que no existe una causalidad directa entre cómo se conforman las salas de clases y el logro de mejores aprendizajes, sino que el mejoramiento pasa por afectar el núcleo pedagógico que corresponde al estudiante y al docente, en presencia de contenidos. Consecuentemente, al momento de juzgar distintas opciones de conformación de cursos, se necesita examinar no solo los logros académicos resultantes, sino también las creencias y prácticas pedagógicas que se asocian a cada opción de agrupamiento. La premisa es alinear la investigación empírica en la materia con los esfuerzos de mejora de las escuelas.

Palabras claves: conformación de cursos, agrupación por habilidades, resultados educacionales, prácticas docentes, creencias, expectativas docentes sobre sus estudiantes.

\begin{abstract}
Abstrac
This paper debates about the link between grouping students into different instructional groups, and learning outcomes. I do a literature review that contrasts different positions and empirical evidence about ability grouping -tracking-. I argue that is not a direct relationship between how students are sorted and grouped, and better academic outcomes, because the improvement is the results of altering the pedagogical core that corresponds to student, teacher and curricular resources. Thus, when alternatives practices of grouping students are put into consideration, it is necessary not only examine educational outcomes, but also beliefs, and teacher practices associated at each option. The mandate is align the empirical research in this topic with schools' improvement efforts.
\end{abstract}

Key words: ability grouping, educational outcomes, teaching method, beliefs, teacher expectations of students

\section{Introducción}

Los responsables de la gestión de las escuelas requieren tomar diversas decisiones organizacionales que no son neutras para los procesos de enseñanza y de aprendizaje. Estas decisiones se refieren a políticas de personal docente, tamaño de los cursos, implementación del currículum prescrito, horarios de clases, así como también a criterios 
para la conformación de cursos de un mismo grado. Respecto a esto último, algunas escuelas pueden optar por agrupar a sus estudiantes según su desempeño académico, conformando cursos relativamente homogéneos. Alternativamente pueden preferir reunir estudiantes de variado rendimiento al interior de una misma clase. Otras escuelas, en cambio, pueden tratar de vincular estudiantes a docentes en función de los estilos de enseñanza o manejo de conducta escolar de los segundos.

En América Latina, la discusión e investigación empírica respecto a los factores que influyen en los aprendizajes, se ha centrado principalmente en aspectos como la calidad docente, la gestión y liderazgo directivo, y la extensión de la jornada escolar. En contraste, son escasos los trabajos que han examinado cómo los estudiantes se distribuyen entre salas de clases de un mismo grado de enseñanza, y los efectos de estos criterios de agrupamiento en los aprendizajes. Esta no es la tendencia en EEUU, donde existe un histórico y extenso debate entre las opciones de agrupar o mezclar a estudiantes según rendimiento académico (Oakes, 1985; Oakes, 1994; Loveless, 1999).

Este artículo pone el foco en los procedimientos de agrupación de estudiantes al interior de las escuelas y su nexo con los resultados de aprendizaje. El argumento central es que las distintas opciones de conformación de cursos, se asocian a diferentes teorías o creencias sobre los procesos de aprendizajes. En ese sentido, se requiere examinar las prácticas pedagógicas al interior de las salas de clases que se vinculan a cada opción de agrupamiento, y no solo los efectos en los aprendizajes. Específicamente se postula que no existe una causalidad directa entre cómo se conforman las salas de clases y el logro de mejores aprendizajes, sino que el mejoramiento pasa por afectar el núcleo pedagógico que corresponde al estudiante y al docente en presencia de contenidos (Elmore, 2010). Con esta lógica el artículo sistematiza estudios en el contexto norteamericano y también de la región, que abordan de manera directa esta temática. El objetivo último es el de relevar la importancia de contar con investigación empírica de carácter mixto que examine los criterios de conformación de cursos al interior de las escuelas, las prácticas pedagógicas que se asocian a las distintas opciones, y los avances académicos resultantes.

\section{Fundamentos del tracking}

El ordenamiento de estudiantes según habilidad en distintos grupos de aprendizajes es una práctica con orígenes históricos en EEUU, cuando a comienzos del siglo pasado la inclusión en el sistema escolar de oleadas de inmigrantes y población de clase trabajadora puso en jaque la capacidad del sistema de cumplir con sus cometidos. Entonces, la separación de estudiantes según habilidades -medidas por test de coeficiente intelectual como el de Binet- se vio como un mecanismo efectivo para organizar el cada vez más diverso sistema escolar, y corregir, a través de la diferenciación curricular, las deficiencias de los estudiantes no tradicionales para integrarlos a la cultura local (Oakes, 1985; Loveless 1999).

En el presente, el uso de test de inteligencia y la diferenciación curricular, ya no son rasgos que caracterizan el modelo escolar norteamericano de escuelas comprensivas, que se basa en la necesidad de brindar una enseñanza común para la construcción de una nación cohesionada. Sin embargo, en la práctica, el ordenamiento de estudiantes según habilidad, persiste en ese país bajo el mandato que tienen las escuelas de alcanzar la eficiencia en la provisión de aprendizajes (Lucas \& Berends, 2002). El término que define esta práctica es el de tracking y su conceptualización difiere a la del contexto europeo, donde tracking toma la forma de sistemas educativos paralelos con currículum diferenciados (al menos uno académico y otro vocacional) a los cuales los alumnos son asignados en función de sus intereses y/o capacidades. Para sus partidarios, el tracking se justifica porque se cree que los estudiantes aprenden mejor cuando están agrupados con pares de similares capacidades o niveles previos de logro (Hallinan, 1994). El tracking permite a los alumnos progresar de acuerdo a sus habilidades y hace posible la adopción de técnicas 
de instrucción adecuadas a sus necesidades particulares. Asimismo, evita que estudiantes menos capaces vean disminuida su autoestima y experimenten actitudes negativas hacia el aprendizaje al estar en contacto y en competencia con pares más talentosos. El tracking reduciría el fracaso escolar, al mismo tiempo que ayudaría a mantener el interés de los estudiantes, porque aquellos más aventajados no se aburren con la participación de los más atrasados académicamente (Ansalone, 2009; Collins \& Gan, 2013). Sin embargo la perspectiva optimista del tracking es rebatida por quienes ponen el foco en la igualdad de oportunidades de aprendizaje, más que en la eficiencia del proceso. Para sus oponentes, las desventajas del tracking se asocian a las menores oportunidades que tendrían los estudiantes rezagados, principalmente porque no se benefician del efecto par de estar con estudiantes de mayor rendimiento, y ser estigmatizados por el resto de sus pares (Oakes, 1985; Goodlad \& Oakes, 1988). Asimismo, se argumenta que para estos grupos de menor rendimiento, los docentes reducirían sus expectativas y se produciría una diferenciación y polarización del currículum.

Es posible inferir, entonces, que la decisión de ordenar a los estudiantes según rendimiento previo en las escuelas, pasaría por la primacía entre directivos y docentes de la creencia que se asocia al efecto directo del tracking: si las clases son homogéneas, entonces los docentes pueden organizar y estructurar mejor su enseñanza al vincular estrechamente sus estrategias de instrucción a los niveles de habilidades de los estudiantes, dejando de lado otras creencias como podrían ser las referidas al efecto par en los aprendizajes. Sin embargo, se debe tener en cuenta que el éxito de esta creencia o teoría de acción del tracking, como la denominaría Elmore (2010), está supeditada a que se entienda exactamente lo que tiene que cambiar en las prácticas pedagógicas para que la conformación uniforme de la clase cumpla con su promesa de generar mejores aprendizajes. Como lo afirma este autor, muchos líderes escolares y profesores pueden creer que es la forma de agrupamiento de los estudiantes la que determina los aprendizajes, olvidando que los elementos que inciden en lo que aprenden sus alumnos se asocian al tipo de contenido a los que estos tienen acceso, y a las técnicas de aprendizaje y expectativas de los docentes. Es decir a lo que sucede en el núcleo pedagógico, y no en las estructuras y procesos que rodean este núcleo.

Lo anterior es concordante con Hallinan (1994) quien señala que efectos negativos del tracking se dan principalmente porque no se cumple el supuesto de adaptar la enseñanza a las necesidades de los estudiantes. En general, los docentes no son capaces o no tienen tiempo de diferenciar la instrucción a los distintos niveles de habilidades o intereses de los estudiantes. Esto hace que sus resultados sean menos eficientes e inequitativos. Junto a ello reconoce que otros efectos negativos se dan por la naturaleza misma de la práctica que distingue y separa estudiantes por consideraciones académicas. El reto entonces es hacer que la implementación del tracking sea consistente con lo que la teoría afirma, y reducir sus rasgos negativos inherentes a su esencia combinándolos con otras políticas o prácticas educativas, como el reconocimiento a los alumnos de bajo desempeño en ámbitos distintos al académico.

Una postura distinta tiene Oakes (1994) quien rebate los planteamientos de Hallinan (1994) señalando que no es posible neutralizar los efectos negativos del tracking ya que no es una práctica organizacional neutra, meramente técnica, sino que está asociada a los valores y creencias de las instituciones o sistemas en las que se implementa. Por lo 
tanto, sus consecuencias no solo deben ser juzgadas en términos de avances cognitivos de los estudiantes, sino que también se deben considerar los efectos sociales y culturales que genera en su entorno. Para esta autora, el tracking crea obstáculos estructurales a los grupos desfavorecidos, impidiendo el logro de objetivos altamente valorados en educación, como el de alcanzar altos niveles de excelencia académica y el de asegurar a todos los estudiantes las mismas oportunidades de alcanzar estos niveles (Oakes, 1985). Sus argumentos, entonces, sobrepasan los procesos y prácticas de los actores escolares, ya que se refieren a cuestiones estructurales y normativas.

\section{Estudios empíricos sobre los efectos del tracking y otras prácticas de agrupamiento}

El tracking ha sido materia de intenso debate en la literatura educacional. El foco se ha centrado en determinar si efectivamente esta práctica es más eficiente que otros mecanismos de agrupamiento, y si beneficia a todos los estudiantes por igual. En un intento por sistematizar el debate acerca de los efectos del tracking en EEUU, Ansalone (2009) agrupa la evidencia empírica en torno a los supuestos que justifican la implementación de esta práctica. Entre ellos: i) todos los estudiantes aprenden mejor si están agrupados con pares de similar nivel de habilidad; ii) el tracking es justo, preciso, y mejora la equidad en el proceso educativo; iii) los estudiantes sufren emocionalmente si son agrupados con estudiantes de mayor habilidad; y iv) los docentes prefieren clases homogéneas porque son más fáciles para enseñar.

Con relación al primer supuesto, el autor indica que existe poca evidencia que afirme que el tracking mejora los aprendizajes de todos los estudiantes. En general la investigación conducida sugiere que el tracking beneficia exclusiva y marginalmente a los estudiantes de los grupos más avanzados, a expensas de los de menor rendimiento.

Respecto al supuesto sobre la justicia del tracking, se concluye que el tracking crea un ambiente de aprendizaje injusto porque los docentes adecuan sus expectativas respecto al desempeño de los estudiantes en función del grupo al cual fueron asignados, lo que favorece los aprendizajes de unos y no de otros. A eso se debe agregar la parcialidad curricular que genera el tracking, al exponer a los estudiantes de menor rendimiento a un currículum disminuido en términos de cantidad y calidad, con relación al brindado a estudiantes de mayor rendimiento.

Por otra parte, la evidencia sobre el impacto del tracking en la autoconfianza de los estudiantes, no es concluyente, por lo que no se confirma el supuesto sobre la desventaja de colocar a los estudiantes de menor rendimiento junto con los más avanzados. Finalmente, respecto a las preferencias docentes de enseñar en clases homogéneas, se señala que los pocos estudios que abordan esta temática encuentran que si bien los docentes prefieren clases con estudiantes de similares niveles de habilidad, sus razones son simplemente de manejo de clase y no académicas. Con todo esto se concluye que el tracking lejos de favorecer la eficiencia de los procesos de aprendizaje de los estudiantes, es una práctica que va en desmedro de la excelencia y equidad en la educación.

Un estudio que encuentra evidencia contraria a la reportada por Ansalone (2009), es el de Collins y Gan (2013) que a partir de un diseño cuasi-experimental, con datos del distrito escolar de Dallas en EEUU, examina cómo la decisión de ordenar estudiantes en distintas clases impacta en los aprendizajes. Sus resultados muestran que agrupar a los estudiantes homogéneamente, según rendimiento académico previo, tiene importantes efectos en el mejoramiento de puntajes en pruebas estandarizadas de matemáticas y lenguaje. La explicación de los autores a este hallazgo es que el "efecto tracking", que permite a los docentes direccionar su enseñanza más eficientemente al tener una clase homogénea, 
es mayor que el "efecto par", que se refiere a la influencia que tiene en el aprendizaje de un estudiante el nivel o calidad de sus compañeros de sala de clases.

Sin embargo, este estudio al igual que otros bajo el mismo diseño, omite examinar las prácticas pedagógicas y los comportamientos particulares de los docentes que se asocian a las estrategias de agrupación de los estudiantes. Tal vez los docentes de las escuelas que hacen tracking tienen altas expectativas para todos los estudiantes, o tal vez estos profesores están adaptando efectivamente su instrucción para satisfacer las necesidades de cada grupo. No es posible identificar la causa de la mejora en los resultados sin examinar antes las prácticas de aula, y los comportamientos de los docentes en cada una de las escuelas que conforman la muestra del estudio.

Un estudio que también entrega evidencia a favor del tracking y que es más explícito en dar cuenta de los mecanismos o condiciones involucradas en las ganancias de aprendizajes es el de Duflo, Dupas y Kremer (2008). Se trata de una investigación experimental realizada en 120 escuelas primarias de Kenia, en las cuales se separa a los estudiantes de primer grado en dos cursos. En 60 escuelas los estudiantes son asignados a los cursos según su rendimiento académico previo, mientras que en las 60 restantes la conformación de los cursos es aleatoria. En ambos tipos de escuela, con y sin tracking, los docentes fueron distribuidos aleatoriamente entre cursos de un mismo centro escolar, al mismo tiempo que tuvieron acceso a material pedagógico apropiado para cada tipo de rendimiento (alto y bajo). Los resultados del experimento muestran que los estudiantes colocados en cursos con pares de similar rendimiento tuvieron mejores avances de aprendizajes, que perduraron más allá de los 18 meses que duró la intervención. Sin duda se trata de un hallazgo en un escenario condicionado que es distinto a los contextos cotidianos donde el tracking se da en la práctica, ya que en general se tiende asignar a los mejores maestros a los cursos más avanzados, al mismo tiempo que los docentes no tienen acceso a material de apoyo pedagógico para distintos niveles de desempeño de sus estudiantes y deben adaptar sus prácticas al estudiante promedio. No obstante, el estudio da cuenta que los aprendizaje de los estudiantes potencialmente pueden mejorar cuando se afecta el núcleo pedagógico. Es decir, cuando las prácticas de agrupamiento según desempeño académico, se complementan con soportes para las estrategias pedagógicas de los docentes.

En Chile, una de las pocas investigaciones que aborda las decisiones de las escuelas respecto a los criterios de conformación de cursos es la de Huepe (2007) quien, a partir de un modelo económico teórico, busca predecir las decisiones de las escuelas privadas respecto del tamaño de sus clases y la composición de sus alumnos. Si bien el resultado que domina el modelo estándar es que separar según habilidad es más eficiente que mezclar, una extensión del modelo que incorpora supuestos sobre la calidad docente y las prácticas pedagógicas arroja resultados ambiguos, ya que bajo ciertas condiciones es óptimo mezclar.

Otro estudio es el de Treviño, Valenzuela y Villalobos (2014) quienes examinan la magnitud, principales factores explicativos y efectos de la segregación académica al interior de las escuelas chilenas. Sus hallazgos revelan que en el sistema chileno, el agrupamiento académico es un proceso extensivo, sobre todo en la secundaria, y que usa distintos mecanismos para lograr una homogenización de los estudiantes, principalmente las notas escolares. Asimismo encuentra que los efectos del agrupamiento académico al interior de las escuelas es negativo, ya que produce una ineficacia en el logro general y una inequidad de los resultados, siendo perjudicados especialmente los estudiantes de rendimiento medio.

A una conclusión distinta llega Wößmann (2005) quien con datos del Estudio Internacional del Progreso en Competencia Lectora (PIRLS, por sus siglas en ingles), examina la relación 
entre familias, escuelas y desempeño de los estudiantes en Colombia y Argentina. Sus resultados dan cuenta que en este segundo país, el currículum centralizado y la conformación de cursos según habilidad al interior de las escuelas, se asocia positivamente con mejores puntajes en esta prueba estandarizada.

Pero el tracking no es la única estrategia de conformación de cursos por habilidad. En EEUU, una estrategia recomendada para atender a los estudiantes de alto rendimiento en escuelas regulares, como alternativa a los eliminados programas de alumnos talentosos, es la agrupación total de estudiantes en cluster (Gentry \& Mac Dougall, 2008). Esta estrategia consiste en dividir a los estudiantes de un mismo nivel en grupos según rendimiento académico (alto, sobre el promedio, promedio, bajo el promedio, bajo y con necesidades especiales) y distribuirlos en diferentes salas de clases de forma tal de disminuir el número de grupos distintos que cada profesor atiende, mientras se mantiene cierta heterogeneidad. Todos los cursos deben tener un grupo de alumnos con rendimiento arriba del promedio, mientras que los alumnos de alto rendimiento son colocados en un mismo salón de clases al igual de aquellos que precisen soporte especial. Para los dos cursos con grupos de estudiantes con rendimientos extremos, los docentes deben ser especialmente capacitados. Asimismo, los alumnos con problemas de comportamiento deben ser distribuidos equitativamente en los distintos cursos. Finalmente, todos los docentes del nivel deben ser involucrados en la definición de los grupos de alumnos según rendimiento y de las listas de clases.

Para Gentry y Mac Dougall (2008), esta estrategia de conformación de cursos ofrece un medio para mejorar el currículum, la instrucción y los aprendizajes, a través del desarrollo del personal docente, el agrupamiento flexible de estudiantes y su colocación en la estructura regular de la escuela. Se trata de una alternativa que además de desafiar a los más avanzados, ubicándolos juntos en una sala de clases, permite a los estudiantes de otras aulas convertirse en lideres académicos, favoreciendo el surgimiento de nuevos talentos. Por otra parte, contribuye a mejorar la atención de los maestros a las necesidades de los estudiantes, al reducir la gama de niveles de rendimiento de los estudiantes en las aulas, y entrenar especialmente a aquellos que atienden tanto a los de más alto rendimiento como los de más bajo. Otra ventaja es la continua evaluación e identificación de fortalezas y debilidades de los estudiantes que demanda esta estrategia de agrupación, superando así al tracking que admite poca o ninguna heterogeneidad al interior de los grupos, además que rigidiza la ubicación de los estudiantes al otorgarles escasa opción de movilidad entre cursos una vez que estos son colocados en ellos.

Los resultados de una evaluación experimental a esta estrategia de conformación de cursos, dan cuenta que después de tres años de su implementación, más estudiantes son clasificados en niveles de rendimiento mayores al promedio y menos en rendimiento bajo, con relación a lo que sucede en escuelas no tratadas (Gentry \& Owen, 1999). En coherencias con estos hallazgos, autores como Hidalgo-Hidalgo (2011) sugieren que la composición de clases óptima para los resultados educacionales es aquella donde los alumnos no se diferencian demasiado en su desempeño académico inicial, pero sí existe una gradualidad entre los extremos. Dicho en otras palabras, donde las clases no son estrictamente homogéneas, sino que hay continuidad entre distintos niveles, no existiendo dos focos de alumnos muy distintos, lo que es perjudicial.

\section{Reflexiones finales}

Hasta aquí se ha dado cuenta de los fundamentos y efectos asociados al tracking y otras estrategias de conformación de cursos como el cluster total de estudiantes. Sin embargo, se debe considerar que no existe una forma de agrupamiento que funcione siempre para todas las escuelas. La mejor estrategia es aquella que está en línea con los contenidos y 
prácticas pedagógicas desplegadas por los docentes, la cultura de la escuela y la creencia de los docentes respecto a sus estudiantes. En la terminología de Elmore (2010) no es el criterio de conformación de clases el que se asocia con mejores aprendizajes, sino la coherencia de la teoría de acción adoptada por los responsables de la gestión escolar y aquello que se gesta en el núcleo pedagógico, conformado por los docentes y los alumnos en la presencia de los contenidos.

Es por ello que, al momento de evaluar los resultados asociados a las prácticas de conformación de cursos en las escuelas, es necesario tomar en consideración la manera y el contexto organizacional en el que estas prácticas son implementadas. Para esto se requiere de diseños mixtos de investigación, que junto con cuantificar efectos y medir diferencias, indaguen también percepciones, creencias y conductas que se asocian a las decisiones organizacionales de las escuelas respecto a la conformación de sus salas de clases.

En los sistemas escolares de América Latina, la alta segregación socioeconómica entre establecimientos educacionales, limita en cierto grado la mixtura de estudiantes en su interior, a diferencia de lo que sucede en sistemas más comprehensivos como el de EEUU. Sin embargo, políticas educativas como las que recientemente se están implementando en Chile, que apuntan a eliminar la selección académica de estudiantes y el copago de las familias en establecimientos financiados con recursos estatales, en el mediano plazo debieran tender a cambiar la composición de los centros escolares. Esto traerá nuevos desafíos a los responsables de la gestión de las escuelas, acentuándose la relevancia de los criterios con los que éstos conformen los diferentes grupos-curso de un mismo nivel de enseñanza, atendiéndose a una población estudiantil más heterogénea. El cómo las distintas opciones de conformación de cursos se asocien a diferentes creencias sobre los procesos de aprendizajes, y las prácticas efectivamente implementadas, serán la clave para que una mayor inclusión en las escuelas, desencadene en una mejora de los resultados académicos de los estudiantes.

\section{Referencias bibliográficas}

Ansalone, G. (2009). Tracking, Schooling and the Equality of Educational Opportunity. Race, Gender \& Class, 16(3/4), 174-184. http://www.jstor.org/stable/41674683?seq=1\#page scan_tab_contents

Collins, C. A., \& Gan, L. (2013). Does Sorting Students Improve Scores? An Analysis of Class Composition (No. w18848). National Bureau of Economic Research. http://www.nber.org/ papers/w18848

Duflo, E., Dupas, P., \& Kremer, M. (2008). Peer effects, teacher incentives, and the impact of tracking: Evidence from a randomized evaluation in Kenya. Cambridge, M.A. http://www.nber.org/papers/w14475

Elmore, R. (2010). Mejorando la escuela desde la sala de clases. Santiago de Chile: Fundación Chile.

Gentry, M., \& Owen, S. V. (1999). An investigation of the effects of total school flexible cluster grouping on identification, achievement, and classroom practices. Gifted Child Quarterly, 43(4), 224-243. http://gcq.sagepub.com/content/43/4/224 
Gentry, M., \& MacDougall, J. (2008). Total school cluster grouping: Model, research, and practice. In J. S. Renzulli \& E. J. Gubbins (Eds.), Systems and models for developing programs for the gifted and talented, 211-234. Mansfield Center, CT: Creative Learning Press.

Goodlad, J., \& Oakes, J. (1988).We must offer equal access to knowledge. Educational Leadership, 45(5), 16-22. http://eric.ed.gov/?id=EJ368820

Hallinan, M. (1994). Tracking: From Theory to Practice. Sociology of Education, 67(2), 79-84. http://www.jstor.org/stable/2112697?seq=1\#page_scan_tab_contents

Hidalgo-Hidalgo, M. (2011). On the optimal allocation of students when peer effects are at work: tracking vs. mixing. SERIEs, 2(1), 31-52. http://link.springer.com/article/10.1007/ s13209-010-0027-2

Huepe, M. (2007). Agrupar o mezclar: decisiones de la escuela en tiempos de escasez. Tesis de Magister en Economía. Pontificia Universidad Católica de Chile. http://economia.uc.cl/ docs/tesis mhuepe.pdf

Loveless, T. (1999). The Tracking Wars: State Reform Meets School Policy. Brookings Institution Press.

Lucas S. \& Berends M. (2002). Sociodemographic, Diversity, Correlated Achievement, and de Facto Tracking. Sociology of Education, 75(4), 328-348.

Oakes, J. (1985). Keeping track: How schools structure inequality (2nd ed.). New Heaven \& London: Yale University Press.

Oakes, J. (1994). More Than Misapplied Technology: A Normative and Political Response to Hallinan on Tracking. Sociology of Education, 67(2), 84-89.

Treviño E., Valenzuela J. y Villalobos C. (2014). Segregación académica y socioeconómica al interior de la escuela. Análisis de su magnitud, principales factores explicativos y efectos. Universidad Diego Portales.

Wößmann, L. (2005). Families, schools, and primary-school learning: Evidence for Argentina and Colombia in an international perspective. World Bank Policy Research Working Paper, (3537). https://openknowledge.worldbank.org/handle/10986/8924?show=full 